\title{
Changes in Muscle Strength and Health Related Quality of Life in Chronic Stroke Subjects after Constraint Induced Movement Therapy (CIMT): A Comparative Study
}

\section{Gopal Nambi S*}

C. U. Shah Physiotherapy College, Surendranagar, Gujarat, India

\section{Abstract}

Background: One of the primary concerns in rehabilitation of stroke patients is to restore motor function in upper limb to perform the myriad of tasks in daily life. Stroke patients begin to compensate for difficult activities by using the unaffected arm, delaying recovery of function in affected arm.

Purpose of the study: The purpose of this study is to find the effectiveness of Constraint Induced Movement Therapy - CIMT in improvement of upper limb function in stroke subjects.

Methods and Design: Experimental Design.

Participants: 30 sub-acute stroke subjects with dominant hand hemiplegia were selected in the study by convenient sampling method. Subjects were randomly assigned into group A and B receiving CIMT \& strengthening exercises respectively for a period of 8 weeks. Prior to the study, all the subjects were assessed using a general assessment Performa and pre treatment ARAT and SS-QOL scores were measured.

Results: There is significant difference between the effectiveness of CIMT and strengthening exercises in improving upper limb function and quality of life in stroke subjects. CIMT is found to be more effective in improving upper limb function than strengthening exercises whereas conventional exercises are more effective in improving the quality of life in stroke subjects.

Conclusion: The clinical findings and the results shows that CIMT was more effective treatment than strengthening exercise in functional outcome and strengthening exercise was more effective than CIMT treatment in Quality of life domain.

Keywords: Stroke; Constraint induced movement therapy; strengthening exercise; Quality of life

\section{Introduction}

Stroke is the $3^{\text {rd }}$ largest cause of death after heart disease and cancer and the most common cause of disability among adults. It is estimated that there are 5.45 million deaths in a year from stroke in the world and over 9 million stroke survivors. The overall prevalence of stroke in the population is estimated to be 47 per 10,000 people [1]. The sequelae of stroke are multi factorial. One of the primary concerns in rehabilitation is to restore motor function in upper limb to perform the myriad of tasks in daily life. Motor tasks require control of muscular force, which becomes compromised in stroke and manifests as impaired coordination, spasticity, and weakness [2].

Stroke patients begin to compensate for difficult activities by using the unaffected arm, delaying recovery of function in affected arm. Many stroke survivors experience impairments such as hemiparesis, spasticity, sensory/perceptual disorders, hemianopsia, dysphasia or cognitive impairments [3]. Most patients regain their walking ability, but between 30 and $60 \%$ are no longer able to use their more affected hand after 3-6 months [4]. Only $11 \%$ to $18 \%$ of those sustaining a severe post stroke upper extremity paresis achieve full upper extremity function [5]. The inability to reach, to grasp and to manipulate objects limits activities and causes particular difficulties to perform daily personal care. Thus, there is a strong need to develop effective armhand treatment methods in stroke rehabilitation.

Various Neuro facilitation approaches have been applied in stroke rehabilitation during the last decades, for example Bobath's neurodevelopment technique, Brunnström's movement therapy and proprioceptive neuromuscular facilitation (PNF) developed by Knott and Voss which was based on the hierarchical theory of motor control. A more recent theory on motor control refers to system's theory, in which a dynamic interplay between perception, cognition, and action systems results in organized and normal movements. In this approach impairments are treated most effectively through repetitive, taskoriented and goal-directed activities [6].

The treatment approach Constraint Induced Movement Therapy - CIMT is based on the theory learned non-use by restraining the unaffected arm by wearing a sling, mitt or plaster cast. The focus of CIMT lies with forcing the patient to use the affected limb by restraining the unaffected one. Intense, repetitive training of the weaker arm is usually given for 90 percent of waking hours 13 hours/day for 2-weeks period. This can be done in the clinic, at home, and wherever else it is safe to do so [7].

A straightforward, yet efficacious, solution to CIMT's limitations has been shorter protocols forcing use of the more affected arm. The most notable example has been modified constraint-induced therapy (mCIMT), which combines structured, half hour, functional practice

*Corresponding author: Gopal Nambi S, C. U. Shah Physiotherapy College, Dudrej Road, Surendranagar, Gujarat, India, Tel: +91 9974757622 9375564344: E-mail: physio_gopal@rediffmail.com

Received March 04, 2014; Accepted June 30, 2014; Published July 30, 2014

Citation: Gopal Nambi S (2014) Changes in Muscle Strength and Health Related Quality of Life in Chronic Stroke Subjects after Constraint Induced Movement Therapy (CIMT): A Comparative Study. Int J Neurorehabilitation 1: 110. doi:10.4172/2376-0281.1000110

Copyright: ( 2014 Gopal Nambi S. This is an open-access article distributed under the terms of the Creative Commons Attribution License, which permits unrestricted use, distribution, and reproduction in any medium, provided the original author and source are credited. 
sessions with restriction of the less affected arm. A basic principle is to keep extending motor capacity a small increment beyond the performance level already achieved [8]. As a result of the patient engaging in repetitive exercises with the affected limb, the brain grows new neural pathways thus improving upper limb function in stroke subjects [9]. CIMT or modifications of CIMT have been applied mainly in chronic stroke patients, when the patients are supposed to have reached a plateau in recovery [10].

The technique has also been applied after sub acute [11] and acute stroke subjects [12]. Different approaches of hand training, such as general task practice (i.e., eating a meal, making coffee, dialing phone numbers and so on) as well as focused-shaping exercises are used in CIMT [13]. It has been suggested that shaping exercises is of outmost importance for improving upper limb motor function and to transfer arm-hand use to real life situations. The shaping technique is defined as a behavioral technique in which movements are approached in small steps of progressively increasing difficulties including verbal feedback. The shaping exercises are similar to ordinary training techniques used by physiotherapists and occupational therapists, but a shaping task is broken down to subtasks, is quantifiable and is repeated many times so small improvements in performance can be measured and systematically made apparent to the patient [14]

According to Page et al., CIMT is not widely viewed by clinicians as a useful therapeutic intervention and considered unfeasible due to patients' concerns about the intensive schedule of treatment. In addition, therapists are concerned about patients' compliance, about safety issues and about clinical resources [15]. To make CIMT clinically adaptable with limited resources as regards therapists, modification of the original concept has been attempted, for example Modified CIMT consisting of 3 times per week for 10 weeks with the intact arm in restraint 5 hours/day for 5 days/week. Two possible mechanisms for the observed effects are believed to be (1) overcoming the learned nonuse of the more affected arm - increased use of the more affected arm and (2) use dependent cortical reorganization.

Mechanisms of brain plasticity include the capacity for neuro chemical, neuro receptor and neuronal structural changes. Normally, many connections between the periphery and the cortex as well as intra cortical connections are probably physiologically silent because of inhibitory influences [16]. Long-lasting immobilization or deprivation of sensory feedback with anesthesia of a body part [17] has been shown to decrease the representational cortical areas, whereas exercises or use of a body part have been reported to enlarged and/or to shift such areas [18]. It has been reported that neural plasticity can occur quickly, and cortical representational changes have been observed after only 5-30 minutes of simple repeated thumb movements [19]. It has been reported that, following rehabilitation of the upper extremity with CIMT [20], the motor output area of the contra lateral (injured) hemisphere expanded [21] in parallel to an improvement in motor function. Interestingly, a new activation in the contra lateral pre motor and motor areas in parallel to an improved hand function has also been observed after CIMT [22].

Many of the traditional perspectives on neuro rehabilitation held that spasticity presented the most significant limitation to recovery of normal motor function. Moreover, because physical exertion was clinically observed to exacerbate spasticity, therapeutic activities using forceful contractions became strictly prescribed for persons with nervous system injury. One prominent approach to treatment of adult hemiplegia thus centered on the concept of managing muscle hypertonia [23], while the general goal of neuro rehabilitation treatment approaches evolved to focus on improving control, and especially the quality of movement. Currently emerging evidence suggests that weakness may be directly responsible for compromised motor function [24]. This premise has motivated research demonstrating that neither effortful activities nor strength training, per se, exacerbate spasticity [25].

Following inactivity and immobilization, almost any vigorous activity will improve strength. Strength training, or progressive resistance training (PRT), generally refers to training with progressively increasing resistive loads beginning at a minimum of 60 percent of that load that can be lifted once (one repetition maximum [1-RM]) [26]. There is a positive relationship between the resistive load and the degree of improvement and remarkable improvements in strength (by 1-RM), functional mobility, and hypertrophy [27].

Accordingly, to induce improvements in strength in hemiparetic persons, studies recommend working at a minimum intensity of 60 percent 1-RM and a maximum of 12 repetitions per set. Outside of these parameters (i.e., loads of less than 60\% 1-RM or greater than 12 repetitions per set), exercise will improve muscular endurance [28].

Upper limb impairment is a major challenge faced by stroke survivors and rehabilitation team. In rehabilitation of hemiplegic upper limb, many studies have shown that CIMT as well as strengthening exercises is effective in improving the functional outcomes. However none of the research studies have compared the effectiveness of both. In this study the effectiveness of CIMT and strengthening exercise is compared. The implications of this study may justify an appropriate and effective treatment technique in improving the upper limb function.

\section{Aim of the Study}

The aim of this study is to compare the effectiveness of CIMT and strengthening exercise in improvement of hemiplegic upper limb function in stroke subjects.

\section{Methods}

An Experimental Design [Comparative study] was used for this study and the subjects are selected by convenient sampling and distributed in two groups by random sampling method. Study was done on sub acute stroke patients who were assessed for cases of first unilateral Cerebro Vascular Accident (CVA). Diagnosis was based on medical notes and routine CT scan reports. Subjects were matched for age, sex and hand dominance for the study. The subjects were explained about the procedure to be done to gain their co-operation and confidence.

Thirty $(\mathrm{n}=30)$ subjects who fulfilled the inclusion and exclusion criteria were included and they were divided into two groups, Group A (Constraint Induced Movement Therapy - CIMT, n=15) and Group B (Strengthening Exercises, $\mathrm{n}=15$ ) and treated for 3 sessions/ week for 4 weeks [7]. Study is done in Out Patient Department of Physiotherapy.

Subjects for the study were selected based on the following inclusion criteria, Stroke between 3 to 12 months post stroke, Preserved cognition (a score of 70 or higher on MMSE), Motor power of at least grade 2 or 3 in the affected upper limb, Adequate balance \& safety while wearing the restraint, Ischemic Stroke, No hemorrhagic lesion, Age group 50 to 70 including both male \& female, No excessive spasticity, defined as a score of three or higher on Modified Ashworth Scale, Patients with dominant hand hemiplegia. Exclusion criteria consists of Treated with intravenous or endovascular therapy, Acute cardiac conditions, Severe osteoporosis, Acute orthopedic or joint injuries, Peripheral nerve 
injuries, fracture, Shoulder hand syndrome, Post surgical conditions, Excessive pain in the affected upper limb, as measured by a score of 4 or higher on a ten point VAS, Participating any of experimental rehabilitation, Taking any of anti spastic drugs, ARAT score below 18.

Patients were assessed when they were alert enough to co operate with testing. The purpose and procedure of the study was explained to the subjects and informed consent was obtained. Demographic data was obtained from the stroke subjects. All the stroke patients were dominant hand hemiplegia. Vital signs were be monitored throughout the treatment. Prior to the study, all the subjects were assessed using a general assessment Performa and pre treatment ARAT and SS-QOL scores were measured with trained physiotherapist who was blinded for the intervention procedure. Post treatment outcome scores were taken at 4 th week \& 8 th week from starting of treatment to compare the results.

Group A received CIMT which was given for 5 hours for 5 days per week for two months. Shaping, a commonly used operant conditioning method in which a behavioural (this case-movement) objective were approached in small steps of progressively increasing difficulty. According to basic principle of CIMT, during therapy sessions, each previously identified skill was practiced for at least 5 minutes as part of the upper-limb program. Because patients were restricted at home, logs were kept to document device use time, as well as activities performed during restraint hours. This component of the program is intended to promote patients' adherence.

For training different tasks were given to patients. E.g. (Combing hair, Eating with spoon \& with hand, Brushing, Shaving, Hand to front \& back pockets, Grooming, Kitchen activities as per need of the patient).

For group B strength was measured by 1 RM. DeLorme reported use of a 1 RM (the greatest amount of weight a subject can lift through the available ROM just one time) as the baseline measurement of a subject's maximum effort. In the clinical setting, a practical, timesaving way to establish a baseline RM for a particular muscle group is for a therapist to select a specific amount of resistance (weight) and document how many repetitions can be completed through the full range before the muscle begins to fatigue. A sign of fatigue is the inability to complete the available ROM against the applied resistance. Strengthening exercises were given at a minimum intensity of $60 \%$ 1-RM and a maximum of 10-12 repetitions given per set. Three sets each of 8 exercises were performed 4 times a week including shoulder, elbow and wrist joint muscles. Grip strengthening exercise was also done. For resistance different sizes of weight cuffs were used. Once patient was able to hold dumbbell safely exercises were started with them.

Conventional therapies were given to both the group A \& group B as follows. Stretching to tight muscles (e.g. calf, hamstring, illio psoas, long finger flexors, pectoralis major), Gait training, Partial squatting exercises, Stair climbing, Different weight bearing exercises, Balance exercises, Pelvic bridging, Prone kneeling exercises.

The statistical analyses were done using software called Statistical Package for Social Science (SPSS 16) for windows. The statistical analyses used were Descriptive analysis to find Means and standard deviation. Inter group comparison was analyzed by using non parametric Mann Whitney U test. Intra group pre \& post values comparison was analyzed by Wilcoxon Sign Rank test.

\section{Results}

\section{Gender Distribution}

Group-A have 9 males (60\%) and 6 females (40\%). Group-B have 7 males $(46.66 \%)$ and 8 females $(53.33 \%)$.

\section{Age Distribution}

Group-A mean age is 57.20 and a S.D \pm 5.88 and Group-B mean age is 59.46 and S D 5.44 .

Inter-group comparison of Pre ARAT score of Group A and B: After analyzing the data with Mann-Whitney Test, the calculated $z$-value is -0.418 and $p$ value is $0.683(p>0.05)$ for ARAT score. The result shows that $p-0.683$ greater than $0.05(p>0.05)$ showing that there is no significant difference between pre values of ARAT scores of Group $\mathrm{A}$ and $\mathrm{B}$. The null hypothesis is accepted and Alternative hypothesis is rejected. Results show the sample homogeneity.

Inter-group comparison of Post ARAT score of Group A and B: After analyzing the data with Mann-Whitney Test, the calculated $\mathrm{z}$-value is -3.044 and $\mathrm{p}$ value is $0.002(\mathrm{p}<0.05)$ for ARAT score. The result shows that $\mathrm{p}-0.002$ less than $0.05(\mathrm{p}<0.05)$ showing that there is significant difference between post values of ARAT scores of Group $\mathrm{A}$ and $\mathrm{B}$. The null hypothesis is rejected and Alternative hypothesis is accepted.

Pre and Post Values of ARAT score of Group A: The Pre Mean ARAT score is 30.53 and after 8 weeks score is 44.8 . In Group-A calculated Z-value is -3.422 and p-value is 0.001 , showing that there is $(p<0.05)$ significant difference between pre and post values of ARAT score of Group A. The null hypothesis is rejected and Alternative hypothesis is accepted.

Pre and Post Values of ARAT score of Group B: The Pre Mean ARAT score is 30.07 and after 8 weeks score is 39.4. In Group-B calculated $\mathrm{Z}$-value is -3.422 and $\mathrm{p}$-value is 0.001 , showing that there is $(p<0.05)$ significant difference between pre and post values of ARAT score of Group B. The null hypothesis is rejected and Alternative hypothesis is accepted.

Inter-group comparison of Pre SSQOL score of Group A and B: After analyzing the data with Mann-Whitney Test, the calculated $\mathrm{z}$-value is -0.873 and $\mathrm{p}$ value is $0.389(\mathrm{p}>0.05)$ for SSQOL score. The result shows that $\mathrm{p}-0.389$ greater than $0.05(\mathrm{p}>0.05)$ showing that there is no significant difference between pre values of SSQOL scores of Group A and B. The null hypothesis is accepted and Alternative hypothesis is rejected. Results show the sample homogeneity.

Inter-group comparison of Post SSQOL score of Group A and B: After analyzing the data with Mann-Whitney Test, the calculated $\mathrm{z}$-value is -2.845 and $\mathrm{p}$ value is $0.004(\mathrm{p}<0.05)$ for ARAT score. The result shows that $\mathrm{p}-0.002$ less than $0.05(\mathrm{p}<0.05)$ showing that there is significant difference between post values of SSQOL scores of Group $\mathrm{A}$ and $\mathrm{B}$. The null hypothesis is rejected and Alternative hypothesis is accepted (Table 1).

Pre and Post Values of SSQOL score of Group A: The Pre Mean SSQOL score is 192.4 and after 8 weeks score is 209.6. In Group-A calculated $\mathrm{Z}$-value is -3.410 and $\mathrm{p}$-value is 0.001 , showing that there is $(\mathrm{p}<0.05)$ significant difference between pre and post values of SSQOL score of Group A. The null hypothesis is rejected and Alternative hypothesis is accepted. 


\begin{tabular}{|c|c|c|c|c|c|c|}
\hline \multirow{2}{*}{ Study variables } & \multirow{2}{*}{ Group } & Baseline & 4 weeks & $\begin{array}{l}\text { Percentage of } \\
\text { Improvement }\end{array}$ & \multicolumn{2}{|l|}{ p-values } \\
\hline & & \multicolumn{3}{|c|}{ Mean value } & Between group & Within group \\
\hline \multirow[t]{2}{*}{ ARAT Score } & A & 30.53 & 44.80 & $66.62 \%$ & \multirow[t]{2}{*}{0.002} & \multirow[t]{2}{*}{0.001} \\
\hline & B & 30.07 & 39.40 & $54.57 \%$ & & \\
\hline \multirow[t]{2}{*}{ SSQOL Score } & A & 192.4 & 209.6 & $38.46 \%$ & \multirow[t]{2}{*}{0.004} & \multirow[t]{2}{*}{0.001} \\
\hline & B & 190.8 & 221.33 & $27.28 \%$ & & \\
\hline
\end{tabular}

Table 1: Pre and post values of ARAT score and SSQOL score of Group-A and Group-B

Pre and Post Values of SSQOL score of Group B: The Pre Mean SSQOL score is 190.8 and after 8 weeks score is 221.3. In Group-B calculated Z-value is -3.411 and p-value is 0.001 , showing that there is $(\mathrm{p}<0.05)$ significant difference between pre and post values of SSQOL score of Group B. The null hypothesis is rejected and Alternative hypothesis is accepted.

\section{Discussion}

The purpose of this study was to compare the effectiveness of CIMT and Strengthening exercise in stroke subjects on the hemiplegic side. The implication of the study may justify the efficacy of CIMT and Strengthening exercise in the improvement of hemiplegic upper limb function. This comparison demonstrated that improvement of hemiplegic upper limb function with an 8 weeks treatment session of CIMT and strengthening exercise in combination with conventional treatment program was efficacious. The outcome measures Action Reach Arm Test (ARAT) and Stroke Specific Quality Of Life (SS-QOL) were used.

The groups were synchronized with age and pretreatment scores of ARAT $(p=0.683)$ and SS-QOL $(p=0.389)$. All participants were treated with standardized programs. Previous studies have shown that there was impairment in the hemiplegic hand function which was treated with CIMT and strengthening exercises and showed that both treatment were effective when they were given individually. Very few studies have compared the effectiveness of these two techniques. But this present study wanted to compare the effectiveness of these two interventions in improvement of upper limb function in the quality of life of sub acute stroke patients.

An 8 weeks treatment was given in both the CIMT and SE group and reassessment was done after $1^{\text {st }}$ month and $2^{\text {nd }}$ month. There was equal improvement in both the groups during the initial days of treatment. But on clinical observation, it was found that after the $1^{\text {st }}$ month reassessment, CIMT shows little more effective in improvement than that of SE. This may be due to the fact that CIMT (task-related training), the practice of goal-directed, functional movements is carried out in a natural environment. In addition, it involves variable practice to help patients derive optimal control strategies for solving motor problems. It was difficult to convince the patients receiving CIMT to apply for 5 hours per day. Moreover few patients (5) had completed full treatment protocol duration and minimized it to 2 to 3 hours per day instead of 5 hours.

This may have an effect on the outcome scores. SE was also found to have improvement in strength without straightforward effects on functional performance; physiologic systems can have associated effects.
Strength training has been demonstrated to decrease depression and improve sleep patterns, influence bone mass, decrease insulin resistance (Type II diabetes), and normalize blood pressure. This may be the reason of showing better scores in SS-QOL in patients receiving SE. Moreover, these improvements in strength appear to transfer to functional improvements.

In previous studies measurement of a single key action such as grip strength for the upper limb or knee extension for the lower limb may be considered a representative surrogate for quantifying motor impairment and disability. During this research it is found that shoulder abductors \& elbow extensors were weaker components as compared to other muscle groups [27]. Therefore using only grip strength \& knee extension as surrogate for quantifying motor impairment may not be accurate. Also some patients $(n=2)$ have reported increase pain and discomfort during SE in the initial few days \& discontinued for 2-3 days but once the pain was relieved they continued with the treatment. Because of these problems it might affect the result \& show lesser improvement in outcome scores.

The functional domain (ARAT score) was found to have more changes than Quality of life domain (SS-QOL score). The result of this study led to the inference that CIMT as well as SE is effective in improving upper limb function \& quality of life. Although both treatments clearly provided additional benefits to patients in the present investigation, it is not possible to identify the precise mechanisms of these effects. While longer term effects of resistance training in hemiparetic persons have not yet been demonstrated, it was found PRT over a period of 24 months has not led to plateaus in increased strength and function in older adults. It would be useful to determine the long term effectiveness of such interventions in future studies [28].

Analysis was done with the baseline data and the post treatment scores. There was significant improvement $(p<0.05)$ in both the groups after an 8 week treatment session with CIMT and SE. This finding suggests that both the treatments are effective for the treatment of improvement in hemiplegic upper limb function. The clinical finding shows that CIMT was more effective treatment than SE in the functional outcome and SE was more effective than CIMT treatment in Quality of life domain. Moreover during clinical examination, it was found that ROM and strength was increased in the SE group than that of mCIMT group with no increase in spasticity which is in accordance with earlier findings.

The results of this study may be applied to a population with a clinical diagnosis of hemiplegia. This study did not include a follow-up period, and, therefore, no conclusions can be drawn about the longterm benefits of the interventions. Because of the short intervention 
and observation periods, one cannot infer that the positive effects of CIMT and SE seen at 8 week of treatment session will lead to prolonged improvements. Further controlled studies with longer observation are required to establish whether these interventions result in long term improvement of symptoms. In the pre and post treatment comparison of ARAT score and SS-QOL score, $\mathrm{p}$ value is $<0.05$ in both the groups. So the null hypothesis got rejected and the alternative hypotheses are accepted.

In conclusion, this experimental comparative study provided evidence to support the use of CIMT and SE regimen in the shortterm management to improve hemiplegic upper limb function. Further studies could focus on the long-term benefits and the relative effectiveness of these treatment regimens compared with other approaches. CIMT shows more improvement in functional domain whereas SE shows more improvement in quality of life domain. Future research can be aimed at combining both the treatment to gain an overall improvement in function as well as quality of life in sub acute stroke patients.

\section{Limitations of the study}

Type of lesion other than ischemic stroke were not considered, Arterials lesions other than that of MCA stroke were not considered, Age group below 50 years were excluded, Acute \& chronic stroke patients were not included and there is limited sample size.

\section{Scope of further study}

Studies based on lower limb function and gait can be performed, Studies may be conducted to find out the deficit in grip strength and different grasps, Studies may be conducted including both the interventions including CIMT \& strengthening exercises to give better improvement in function by increasing strength.

\section{Conclusion}

The study led to the conclusion that both Constraint Induced Movement Therapy and Strengthening Exercise are effective in improving upper limb function and quality of life in stroke subjects. There is significant difference between the effectiveness of Constraint Induced Movement Therapy and Strengthening Exercise in improving upper limb function and quality of life in stroke subjects. CIMT is found to be more effective in improving upper limb function than SE whereas SE is more effective in improving the quality of life in stroke subjects.

\section{Acknowledgement}

Hereby we thank all the subjects and contributors for their contribution towards the successful completion of this project.

\section{References}

1. O'Sullivan, Schmitz (2001) Physical Rehabilitation: Assessment and Treatment. Stroke. 4th ed. New Delhi, Jaypee Brothers.

2. Katz RT, Rovai GP, Brait C, Rymer WZ (1992) Objective quantification of spastic hypertonia: correlation with clinical findings. Arch Phys Med Rehabil 73: 339-347.

3. Gresham GE, Duncan PW, Stason WB (1995) Post-stroke rehabilitation; Clinical practice guideline. AHCPR; 16.

4. Dobkin $\mathrm{BH}(2005)$ Clinical practice. Rehabilitation after stroke. N Engl J Med 352: 1677-1684.

5. Kwakkel G, Kollen BJ, van der Grond J, Prevo AJ (2003) Probability of regaining dexterity in the flaccid upper limb: impact of severity of paresis and time since onset in acute stroke. Stroke 34: 2181-2186.
6. Shumway-Cook A, Woollacott MH (2001) Motor control. Theory and practical applications. Lippincott Williams \& Wilkins.

7. Blanton S, Wolf SL (1999) An application of upper-extremity constraint-induced movement therapy in a patient with subacute stroke. Phys Ther 79: 847-853.

8. Wolf SL, Winstein CJ, Miller JP, Taub E, Uswatte G et al. (2006) Effect of Constraint-Induced Movement Therapy on Upper Extremity Function 3 to 9 Months After Stroke: The EXCITE Randomized Clinical Trial. JAMA 296: 20952104.

9. Porter MM (2000) Resistance training recommendations for older adults. Top Geriatric Rehabil 15: 60-69.

10. Brogårdh C, Sjölund BH (2006) Constraint-induced movement therapy in patients with stroke: a pilot study on effects of small group training and of extended mitt use. Clin Rehabil 20: 218-227.

11. Sinkjaer T, Magnussen I (1994) Passive, intrinsic and reflex-mediated stiffness in the ankle extensors of hemiparetic patients. Brain $117: 355-363$.

12. Ploughman $M$, Corbett $D$ (2004) Can forced-use therapy be clinically applied after stroke? An exploratory randomized controlled trial. Arch Phys Med Rehabil 85: 1417-1423.

13. Taub E, Uswatte G, King DK, Morris D, Crago JE, et al. (2006) A placebocontrolled trial of constraint-induced movement therapy for upper extremity after stroke. Stroke 37: 1045-1049.

14. Morris DM, Crago JE, Deluca SC, Pidikiti RD, Taub E (1997) Constraint-induced movement therapy for moter recovery after stroke. NeuroRehabilitation 9: 2943.

15. Sterr A, Freivogel S (2003) Motor-improvement following intensive training in low-functioning chronic hemiparesis. Neurology 61: 842-844.

16. Wall PD (1977) The presence of ineffective synapses and the circumstances which unmask them. Philos Trans R Soc Lond B Biol Sci 278: 361-372.

17. Rossini PM, Rossi S, Tecchio F, Pasqualetti P, Finazzi-Agrò A, et al. (1996) Focal brain stimulation in healthy humans: motor maps changes following partial hand sensory deprivation. Neurosci Lett 214: 191-195.

18. Elbert T, Pantev C, Wienbruch C, Rockstroh B, Taub E (1995) Increased cortical representation of the fingers of the left hand in string players. Science 270: $305-307$

19. Classen J, Liepert J, Wise SP, Hallett M, Cohen LG (1998) Rapid plasticity of human cortical movement representation induced by practice. J Neurophysio 79: $1117-1123$.

20. Cicinelli P, Traversa R, Rossini PM (1997) Post-stroke reorganization of brain motor output to the hand: a 2-4 month follow-up with focal magnetic transcranial stimulation. Electroencephalogr Clin Neurophysiol. 105: 438-450.

21. Wittenberg GF, Chen R, Ishii K, Bushara KO, Eckloff S, et al. (2003) Constraintinduced therapy in stroke: magnetic-stimulation motor maps and cerebral activation. Neurorehabil Neural Repair 17: 48-57.

22. Kim YH, Park JW, Ko MH, Jang SH, Lee PK (2004) Plastic changes of motor network after constraint-induced movement therapy. Yonsei Med J 45: 241 246.

23. Bobath B (1990) Adult hemiplegia: Evaluation and treatment. Oxford: Butterworth- Heineman.

24. Bohannon RW (1989) Selected determinants of ambulatory capacity in patients with hemiplegia. Clin Rehabil 3: 47-53.

25. Fowler EG, Ho TW, Nwigwe Al, Dorey FJ (2001) The effect of quadriceps femoris muscle strengthening exercises on spasticity in children with cerebral palsy. Phys Ther 81: 1215-1223.

26. Enoka RM (1994) Neuromechanical basis of kinesiology. Champaign,IL: Human Kinetics; 1994.

27. Porter MM (2000) Resistance training recommendations for older adults. Top Geriatr Rehabil 15: 60-69.

28. Patten C, Lexell J, Brown HE (2004) Weakness and strength training in persons with poststroke hemiplegia: rationale, method, and efficacy. J Rehabil Res Dev 41: 293-312. 